\title{
Network pharmacology and chemoproteomics unveil the mechanism of Total Flavonoid Aglycones Extract inhibiting EMT in A549 cell line
}

\author{
Hui-Juan Cao ${ }^{1 \dagger}$, Wei Zhou ${ }^{2 \dagger}$, Xiao-Le Xian ${ }^{1 \dagger}$, Shu-Jun Sun ${ }^{3}$, Pei-Jie Ding ${ }^{1}$, Chun-Yu Tian ${ }^{1}$, Fu- \\ Ling Tian ${ }^{1}$, Chun-Hua Jiang ${ }^{1}$, Ting-Ting $\mathrm{Fu}^{1}$, Shu Zhao ${ }^{1}$, Jian-Ye Dai ${ }^{2,4 *}$ \\ 1. Traditional Chinese Medicine College, North China University of Science and Technology, \\ Tangshan 063210, China \\ 2. School of Pharmacy, Lanzhou University, Lanzhou 730000, China \\ 3. School of biology and food engineering, Fuyang Normal University, Fuyang 236037, China \\ 4. State Key Laboratory of Natural Medicines, China Pharmaceutical University, Nanjing \\ 210009, China
}

$\dagger$ These authors have contributed equally to this article

* Correspondence:

Corresponding Author: Jian-Ye Dai

daijy@1zu.edu.cn

Number of words:4057; Number of Figures:4; Number of tables:1.

Keywords: Total Flavonoid Aglycones Extract, Epithelial-mesenchymal transition, Network pharmacology, Stable isotope dimethyl-labeled proteomics technology, TWIST1, PI3K/Akt signaling pathway

\begin{abstract}
Non-small cell lung cancer is a worldwide disease with high morbidity and mortality, the epithelialmesenchymal transition process promotes cancer cell migration and invasion, leading to NSCLC metastasis. Total Flavonoid Aglycones Extract isolated from Scutellaria baicalensis was reported to inhibit tumor growth and induce apoptosis. In this study, combining network pharmacology and stable isotope dimethyl-labeled proteomics technology, we found the inhibitory effect of TFAE on the EMT process of A549 cells, and established the relationship between TFAE, PI3K/Akt signaling pathways, TWIST1, and glycolysis pathway. LY294002 was incubated with A549 cells to inhibit PI3K/Akt signaling pathway, and small interfering RNA method was applied to knock down the TWIST1 gene in A549 cell. Compared with normal cells, LY294002-treated/ TFAE-treated/ TWIST1-silenced / TFAE and siTWIST1-treated cells showed poor migration and invasion capabilities, increased expression of epithelial markers and decreased expression of mesenchymal markers. All the results suggested that TFAE inhibits PI3K/Akt pathway and TWIST1 and then inhibits glycolysis pathway, thereby inhibiting the EMT process of A549 cells.
\end{abstract}

\section{Introduction}

Lung cancer is a worldwide disease with high morbidity and mortality. According to World Health Organization(2020), there were 2.207 million new lung cancer patients worldwide in 2020, less than breast cancer; and lung cancer ranked first in the global causes of death. Among the histological subtypes of lung cancer collectively, non-small cell lung cancer (NSCLC) accounts for $80 \%-85 \%$; and most lung cancer patients are diagnosed at advanced stages, so traditional chemotherapy and 
radiotherapy have limited efficacy(MPH et al., 2017). NSCLC microenvironment has limited nutrition, so cancer cells often obtain the energy and substances required for proliferation and cell growth through the "Warburg Effect" glucose metabolism changes(Gatenby and Gillies, 2004;LopezLazaro, 2008), that is, cancer cells tend to produce energy through glycolysis rather than oxidative phosphorylation(Heiden et al., 2009; Muz et al., 2015;Eales et al., 2016). However, the metabolic change leads to further tumor cell growth, aggravation of epithelial-mesenchymal transition (EMT) process and resistance to treatment. During the treatment of NSCLC, $40 \%$ of patients have metastases(Keith, 2017), and the main biological process is EMT (Tulchinsky et al., 2018). In EMT process, cells gradually become spindle-shaped, losing the unique epithelial characteristics, intercellular adhesion and cytoskeletal structure of epithelial tissue, and possessing polarity, individual migration ability and invasion ability(Trott and Olson, 2010). Cellular EMT process is related to TGFR, Wnt/ $\beta$-Catanin, PI3K/Akt, Ras, MAPK, NF- $\kappa$ B pathways, and the transcription factors involved are TWIST (TWIST1 and TWIST2), Slug, ZEB (ZEB1 and ZEB2), Snail et al(T and Benjamin, 2011; Otsuki et al., 2018).EMT transcription factors promote the development of drug resistance in tumor therapy and may become new targets for tumor therapy(Jente et al., 2018; Tieju et al., 2020) . In the process of EMT, the activated PI3K/Akt pathway promotes glucose uptake and glycolysis(L, 2010; Tennant et al., 2010), and phosphorylated Akt can phosphorylate TWIST1, then up-regulates the TGF $\beta$-Smad pathway, which further promotes the EMT process (Gongda et al., 2012). TWIST1 was known to bind to the E-box sequence of E-cadherin and inhibited the expression of E-cadherin to induce EMT in a variety of tumors (Hong et al., 2016; Qing-Qing et al., 2016;Ding et al., 2019).Inhibition of TWIST1 was known to induce growth inhibition and apoptosis of EGFRmutant NSCLC cells(Yochum et al., 2019).

Many traditional herbals and their extracts have been reported to have therapeutic effects on cancer, including Scutellaria baicalensis (P et al., 2000;Dorota et al., 2015). The main components of Scutellaria baicalensis are flavonoids, but the bioavailability is limited due to the high polarity after formation of glycosides. Therefore, hydrolysis of flavonoid glycosides into aglycones improves their bioavailability. Total Flavonoid Aglycones Extract (TFAE) isolated from Scutellaria baicalensis was reported to inhibit tumor growth and induce apoptosis (Yang et al., 2016). Its main components were baicalein, wogonin, and oroxylin-A, and their proportions were $41.4 \%, 13.3 \%$, and $8.2 \%$, respectively (Figure 1A). Considering the high metastasis of NSCLC, we studied the mechanism of TFAE inhibiting EMT of A549 cells.

In this study, TFAE was prepared according to the ratio of baicalein, wogonin, and oroxylin-A in the extracted total flavonoids of Scutellaria baicalensis, to explore its effect on the EMT process of A549 cells. Network pharmacology was performed to explore connections between TFAE and lung cancer, and stable isotope dimethyl-labeled proteomics was performed to find out the pathways and proteins affected by TFAE. After targets screening, LY204002 was incubated with A549 cells to inhibit PI3K/Akt signaling pathway, and small interfering RNA (siRNA) method was applied to knock down the TWIST1 gene in A549 cell. Wound healing assay and cell invasion assay were applied to monitor cell migration and invasion ability, and western blot was applied to detect the expression of EMT-related proteins. Cell experiments results showed that TFAE inhibited the EMT process of A549 cells by inhibiting PI3K/Akt pathway and TWIST1. Combined with the proteomics results, we believed that, when the PI3K/Akt pathway was inhibited, TWIST1 would be reduced. Then the expression of PKM, ALDOA, and GPI genes were affected, thereby inhibiting glycolysis and pentose phosphate signaling pathways, which finally inhibited the EMT process of A549 cells. 
Original Research.

\section{Materials and Methods}

\subsection{Cell culture}

The A549 cells was obtained from the Cell Resource Center, Peking Union Medical College, and were cultured at $37{ }^{\circ} \mathrm{C}$ under $5 \% \mathrm{CO}_{2}$ in Dulbecco's modified Eagle's medium (DMEM; Sigma, cat\#D6429) supplemented with 10\% (vol./vol.) fetal bovine serum (FBS; Gibco, cat\#10270-106), and $1 \%$ (vol./vol.) penicillin-streptomycin (PS; Gibco, cat\#15140-122). Culture medium was refreshed and the cells were passaged when the confluency was $80 \%$.

\subsection{Preparation of cisplatin, LY294002 and TFAE}

4mg/mL cisplatin stock solution, 20mM LY294002(AbMole, cat\#M1925) stock solution and $204.8 \mathrm{mg} / \mathrm{mL}$ TFAE solution were prepared by DMSO. For TFAE, the three main components and their mass percentages are baicalein (41.4\%), wogonin (13.3\%), and oroxylin-A (8.2\%). Weigh the amount of each TFAE component according to their mass percentage, then mix the three compounds, and dissolve them to the desired volume with DMSO.

\subsection{Wound healing assay and cell invasion assay}

For wound healing assay: $1.7 * 10^{7}$ A549 cells were plated in six-well plates. 7 hours later, the cells formed a single layer with nearly $100 \%$ confluency. A linear wound was made by scraping the confluent cell layer with a $200 \mu \mathrm{L}$ tip. Cells were washed with PBS for 3 times to remove detached cells and debris. Cells were incubated with complete medium with or without TFAE, Cisplatin, or LY294002 for $24 \mathrm{~h}$. At 0 and 24h after scratching, 3 fields of view for each well were select randomly to take pictures. Image $J$ software were used to scan the scratch areas and to calculate the wound healing area. Wound healing rate= (wound area at $0 \mathrm{~h}-$ wound area at $24 \mathrm{~h}$ )/ wound area at $0 \mathrm{~h}$ $* 100 \%$.

For cell invasion assay: the transwell containing polycarbonate membranes with $8 \mu \mathrm{m}$ pore size was precoated with $50 \mu \mathrm{L}$ of Matrigel $(1.0 \mathrm{mg} / \mathrm{mL})$ and incubated at $37^{\circ} \mathrm{C}$ overnight. $1.0^{*} 10^{6} \mathrm{~A} 549$ cells and TFAE $(3.2,6.4,12.8 \mu \mathrm{g} / \mathrm{mL})$ in serum-free DMEM medium were plated in the upper chamber, followed by $150 \mu \mathrm{L}$ of serum-supplemented DMEM medium in the lower chamber. After $24 \mathrm{~h}$ of incubation, the upper chamber was taken out and washed by wash buffer for 2 times, then was transferred to test board containing $100 \mu \mathrm{L}$ Cell Dissociation Solution/Calcein-AM. After 1h of incubation, the fluorescence value was detected at $485 \mathrm{~nm}$ excitation wavelength and $520 \mathrm{~nm}$ emission wavelength. Cell invasion rate $=$ (number of invaded cells in experimental wells/ number of invaded cells in control wells) $* 100 \%$.

\subsection{Western blot}

The A549 cells treated under different conditions were collected after washed by PBS for three times and centrifugation at $3000 \mathrm{rpm}$ for 3 minutes. The cells were lysed in RIPA Lysis Buffer (CWBIO, cat\#CW2333S) containing EDTA-free cOmplete(Roche, cat\#4693132001) with sonication on ice. The cell lysates were collected by centrifugation $\left(150^{*} 100 \mathrm{rpm}, 20 \mathrm{~min}\right)$ at $4^{\circ} \mathrm{C}$. The protein concentration was detected by PierceTM BCA Protein Assay Kit (Thermo Fisher Scientific, cat\#23225) and adjusted to $2 \mathrm{mg} / \mathrm{mL} .20 \mu \mathrm{g}$ of the lysates were separated by $10 \% \mathrm{SDS}$-PAGE and then transferred onto a PVDF membrane in an ice bath at $300 \mathrm{~mA}$ for $1 \mathrm{~h}$. The membrane were blotted with $5 \%$ nonfat milk in TBST at RT for $1 \mathrm{~h}$ and then incubated with the following antibodies at $4^{\circ} \mathrm{C}$ 
overnight: TWIST1(Cell Signaling Technology, cat\#46702S), Snail(Cell Signaling Technology, cat\#3879), E-cadherin(Cell Signaling Technology, cat\#3195),Vimentin(Cell Signaling Technology, cat\#5741), N-cadherin(Santa, cat\#Sc-59987), AKT(Cell Signaling Technology, cat\#4685), pAKT(Cell Signaling Technology, cat\#4060), $\beta$-Actin(Proteintech, cat\#20536-1-AP). Then the membrane was incubated with secondary antibodies at RT for $1 \mathrm{~h}$. The secondary antibodies are as follows: Goat anti-Rabbit IgG (Proteintech, cat\#SA00001-2), Goat anti-mouse IgG (Proteintech, cat\#SA00001-1). Finally, the immunoreactive bands were visualized by the Clarity ${ }^{\mathrm{TM}}$ Western ECL Substrate (BIO-RAD, cat\#1705061) using the Tanon4600 Automatic chemiluminescence image analysis system (Tanon, Shanghai, China).

\subsection{Transfection with siRNA}

$6.0 * 10^{5}$ A549 cells were plated in six-well plates. 7 hours later, the cells with nearly $70 \%$ confluency were transfected with TWIST1 siRNA. The siRNA sequences were as follows: TWIST1(sense:5'CAAGAUUCAGACCCUCAAGTT-3', antisense:5'-CUUGAGGGUCUGAAUCUUGTT-3'); GAPDH (sense:5' - AATGGGCAGCCGTTAGGAAA -3', antisense: 5'-

TGAAGGGGTCATTGATGGCA -3'). A siRNA nonspecific control was also used in this study. SiRNA sequences were designed and synthesized by GenePharma (Shanghai, China). The interference was performed based on the protocol of Lipofectamine ${ }^{\circledR} 3000$ (Thermo Fisher Scientific, cat\#L3000150). The silencing efficiency was examined by qRT-PCR and western blot.

\subsection{Quantitative real-time PCR (qRT-PCR)}

Total RNA was extracted and purified with Eastep ${ }^{\circledR}$ Super Total RNA Extraction Kit (Promega, cat\#LS1040) and used as the cDNA synthesis template for reverse transcription with the RevertAid First Strand cDNA Synthesis Kit (Thermo Scientific, cat\#k1622). The reverse transcription conditions were as follows: $42^{\circ} \mathrm{C} 30 \mathrm{~min} ; 85^{\circ} \mathrm{C} 10 \mathrm{~min}$. qRT-PCR was performed by MX3000P(Agilent) using UltraSYBR Mixture (Low ROX) (CWBIO, cat\#CW2601), reaction conditions were $: 95^{\circ} \mathrm{C} 3 \mathrm{~min} ; 95^{\circ} \mathrm{C} 15 \mathrm{~s} ; 55^{\circ} \mathrm{C} 30 \mathrm{~s}, 72^{\circ} \mathrm{C} 30 \mathrm{~s} ; 40$ cycles. The primers were as follows: TWIST1(forward: 5'-TCGGACAAGCTGAGAGCAAGATTCA-3', reverse: 5' TCCATCCTCCAGACCGAGAAGG -3'), GAPDH (forward: 5'CATGAGAAGTATGACAACAGCCT-3', reverse: 5'- AGTCCTTCCACGATACCAAAGT-3'). CT values were used to evaluate the relative mRNA expression by $2-\Delta \Delta \mathrm{Ct}$ method and $\beta$-Actin served as an internal control. The primers were designed and synthesized by GenePharma (Shanghai, China).

\subsection{Protein in-solution digestion and dimethyl labeling}

A549 cells were grown to $80 \%$ confluence in $15 \mathrm{~cm}$ dish. A549 cells were treated with $102.4 \mu \mathrm{g} / \mathrm{mL}$ TFAE for $24 \mathrm{~h}$. The cells were collected after washed by PBS for three times and centrifugation at $3000 \mathrm{rpm}$ for 3 minutes. The cells were lysed in 0.1\% Triton X-100(Sigma-Aldrich, cat\#T8787)100mM TEAB (Sigma-Aldrich, cat\#T7408) containing EDTA-free cOmplete with sonication on ice. The cell lysates were collected and the protein concentration was detected by PierceTM BCA Protein Assay Kit. $10 \mu \mathrm{L}$ cell lysates $(3 \mathrm{mg} / \mathrm{mL})$ were reacted with $30 \mu \mathrm{L} 8 \mathrm{M}$ urea and $2 \mu \mathrm{L} 200 \mathrm{mM}$ DTT at $65^{\circ} \mathrm{C}$ for $15 \mathrm{~min}$ in the dark. Then $2 \mu \mathrm{L} 400 \mathrm{mM}$ iodoacetamide was added to react in light for $30 \mathrm{~min}$ at $35^{\circ} \mathrm{C}$. $2 \mu \mathrm{L} 200 \mathrm{mM}$ DTT was added to react with the remaining iodoacetamide in light for $15 \mathrm{~min}$ at $65^{\circ} \mathrm{C} .100 \mu \mathrm{L} 100 \mathrm{mM}$ TEAB, $2 \mu \mathrm{L} 0.2 \mu \mathrm{g} / \mu \mathrm{L}$ trypsin (Promega, cat\#V528A) and $1.5 \mu \mathrm{L} 100 \mathrm{mM}$ $\mathrm{CaCl} 2$ were added and the trypsin digestion was performed at $37^{\circ} \mathrm{C}$ overnight. For dimethyl labeling: for the control("Light") group, the peptides were reacted with $6 \mu \mathrm{L} 4 \% \mathrm{CH} 2 \mathrm{O}$ (Sigma-Aldrich, cat\#F1635) and 6 $\mu \mathrm{L}$ 0.6M NaBH3CN (Sigma-Aldrich, cat\#42077); for the TFAE ("Heavy") group, 
the peptides were reacted with $6 \mu \mathrm{L} 4 \%{ }^{13} \mathrm{CD}_{2} \mathrm{O}$ (Sigma-Aldrich, cat\#596388) and $6 \mu \mathrm{L} 0.6 \mathrm{M}$ $\mathrm{NaBD}_{3} \mathrm{CN}$ (Sigma-Aldrich, cat $\# 190020$ ). The reaction was incubated at $22^{\circ} \mathrm{C}$ for $1 \mathrm{~h}$. The reaction was quenched by $24 \mu \mathrm{L} 1 \%$ ammonia and $12 \mu \mathrm{L}$ formic acid. The "Light" and "Heavy" samples were combined and the desalination was performed by PierceTM C18 Tips (Thermo Scientific, cat\#87782).

\subsection{LC-MS/MS analysis.}

Samples were analyzed by LC-MS/MS on Q Exactive series Orbitrap mass spectrometers (Thermo Fisher Scientific) coupled with EasyNano-LC. In positive-ion mode, full-scan mass spectra were acquired over the $\mathrm{m} / \mathrm{z}$ ratio from 350 to 1800 using the Orbitrap mass analyzer with mass resolution of 7000. MS/MS fragmentation is performed in a data-dependent mode, of which the TOP 20 most intense ions are selected for MS/MS analysis with a resolution of 17500 using collision mode of HCD. Other important parameters: isolation window, $2.0 \mathrm{~m} / \mathrm{z}$ units; default charge, 2+; normalized collision energy, 28\%; maximum IT, 50ms; and dynamic exclusion, 20.0s. LC-MS/MS data was analyzed by ProLuCID with static modification of cysteine $(+57.0215 \mathrm{Da})$. The isotopic modifications (28.0313 and 34.0631 Da for light and heavy labeling, respectively) are set as static modifications on the $\mathrm{N}$-terminal of a peptide and lysines. The ratios of reductive dimethylation were quantified by the CIMAGE software. Proteins with an average ratio(Light/Heavy) above 1.5 were selected for further KEGG analysis.

\subsection{Network pharmacology and molecular docking analysis}

In this study, based on network pharmacology, the common mechanism of TFAE in the treatment of lung cancer was studied. The active ingredients and related targets of TFAE were integrated from TCMSP, BATMAN-TAM, STP and Pubchem databases. The standard names of these targets were united by UniProt database. Targets of lung cancer were enriched through GeneCards, NCBI(Gene), Therapeutic Target Database, and DisGeNET(v7.0) databases. Then the intersection targets of TFAE and disease were obtained. The STRING network and the Cytoscape 3.7.2 were used to construct Protein-Protein-Interaction (PPI) network, the DAVID database was used to perform KEGG analysis. Then Cytoscape 3.6.1 was used to build "Ingredient-Target-Signal Pathway" network. After confirming the key protein and molecules, their 3D structures were obtained from RCSB PDB database and ZINK database, respectively. In AutoDock Tools, hydrogen atoms were added to, while water molecules and co-crystallized ligands were removed from the protein and molecules. Glide was selected to dock according to the previously reported active amino acid sites. The docking was performed by the AutoDock Vina software(Trott and Olson, 2010). And PyMOL was used to visualize various conformations of the ligand and its different binding interactions with the receptor.

\subsection{Statistical analysis.}

Student's t-test was used to compare experimental data. We analyzed the data in GraphPad Prism (GraphPad Software), using the unpaired, two-tailed t-test module. Statistical significance was considered when a $\mathrm{p}$ value was below 0.05 . ${ }^{*} p<0.05 ; * * p<0.01 ; * * p<0.001$. N.S. not significant.

\section{$4 \quad$ Results}

\subsection{TFAE inhibits EMT process of A549 cells}

To explore whether TFAE affects the EMT process of lung cancer, A549 cells were treated with TFAE $(3.2,6.4,12.8 \mu \mathrm{g} / \mathrm{mL})$ for 48 hours. Compared to the non-administered(control) group, the 
invasion rate of cells treated by TFAE concentration-dependently decreased (Figure 1B). In addition, TFAE dose-dependently inhibited the expression of Twist, Snail, p-AKT and N-cadherin proteins, while increased the expression of E-cadherin (Figure 1C). Compared with the wound area at 0 hours, the wound caused by tips were dose-dependently healed by TFAE (Figure 1D), which means TFAE inhibited the migration of A549 cells.
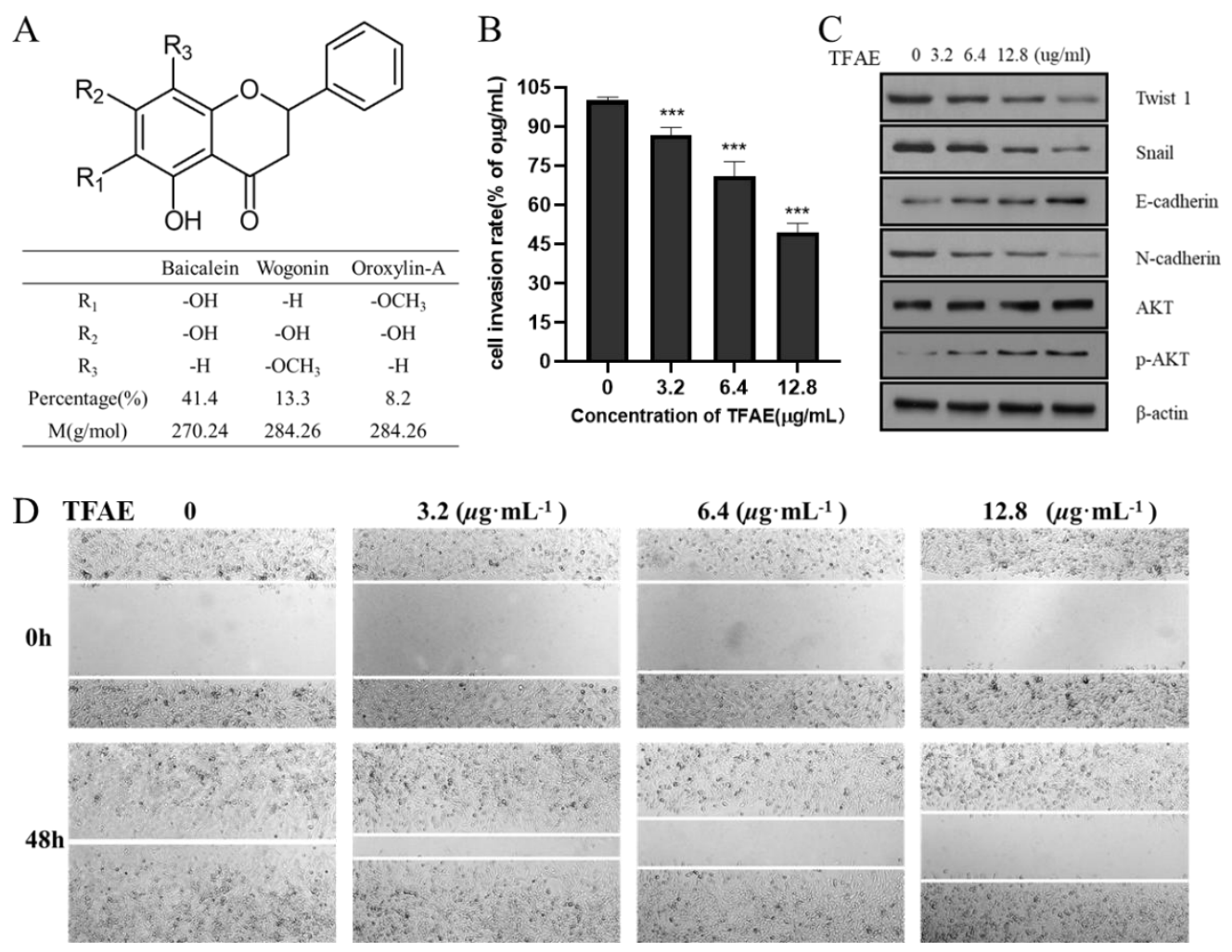

FIGURE 1 | TFAE inhibits EMT process of A549 cells. (A) The structure and proportion of the main components of TFAE. (B) TFAE inhibits A549 cell invasion ability. (C) Effects of different concentrations of TFAE on the expression of EMT-related proteins. (D) TFAE inhibits A549 cell migration. Statistical differences were determined by a two-sided Student's t-test; Compared with $0 \mu \mathrm{g} / \mu \mathrm{L}$ TFAE. ${ }^{* * *} P<0.001$; ${ }^{* *} P<0.01 ;{ }^{*} P<0.05$.

\subsection{TFAE inhibits PI3K/Akt pathway in EMT process of A549 cells}

The above results indicated that TFAE exerted a concentration-dependent inhibitory effect on the EMT process of A549 cells. To explore the connection between TFAE and lung cancer, network pharmacology analysis was performed. Three active ingredients and 65 related targets of TFAE were integrated from TCMSP, BATMAN-TAM, STP and Pubchem databases. 22474 targets of lung cancer were enriched through GeneCards, $\mathrm{NCBI}(\mathrm{Gene})$, Therapeutic Target Database, and DisGeNET(v7.0) databases. Venn diagram showed the intersection of 62 TFAE and lung cancer targets (Figure 2A). STRING network and Cytoscape 3.7.2 were used to construct Protein-ProteinInteraction (PPI) network (Figure 2B), AKT1 was the central protein. DAVID database was used to perform KEGG analysis, TFAE regulated pathways with $\mathrm{P}<0.01$ were as follows: TNF signaling pathway, p53 signaling pathway, PI3K/Akt signaling pathway, VEGF signaling pathway, HIF-1 signaling pathway, MAPK signaling pathway (Figure 2C). In tumor cells, PI3K/Akt signaling pathway is highly activated and directly regulates the expression of TWIST1 and tumor metastasis(Gongda et al., 2012). To investigate whether TFAE affects the EMT process of A549 cells 
by acting on PI3K/Akt signaling pathway, we incubated A549 cells with the PI3K/Akt pathway inhibitor, LY294002. In this part, we used cisplatin as positive control.

Compared with normal cells, PI3K/Akt pathway-suppressed A549 cells showed poor migration and invasion capabilities, which was similar to the TFAE group and cisplatin group (Figure 2D, E).

Moreover, similar to cells given TFAE or cisplatin, PI3K/Akt-suppressed A549 cells showed increased expression of E-cadherin and decreased expression of Vimentin, N-cadherin, TWIST1 and p-AKT (Figure 2F). Network pharmacology results showed that TFAE acted on PI3K/Akt signaling pathway, while cell experiment results showed that inhibition of PI3K/Akt signaling pathway caused inhibition of EMT process. Therefore, we believe that TFAE inhibits the EMT process of A549 cells by inhibiting the PI3K/Akt signaling pathway.
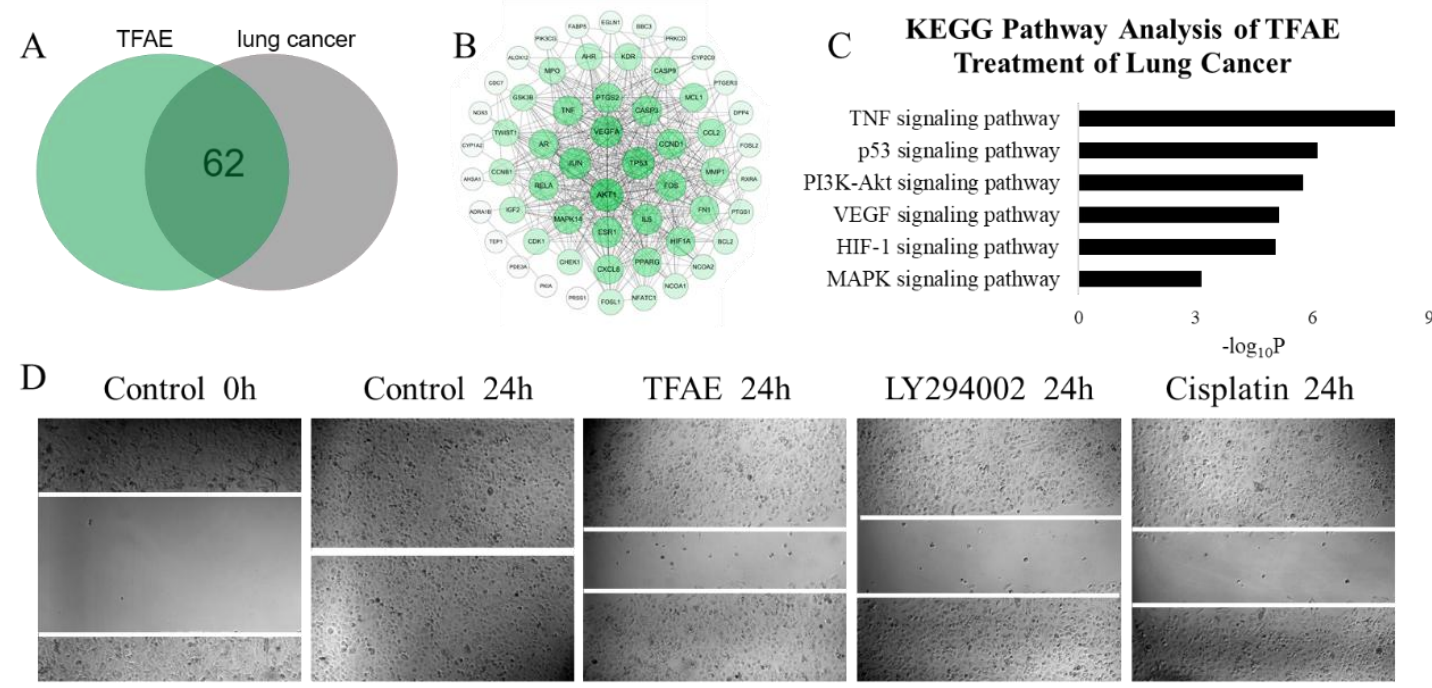

TFAE $24 \mathrm{~h}$

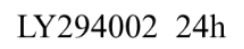

Cisplatin $24 \mathrm{~h}$
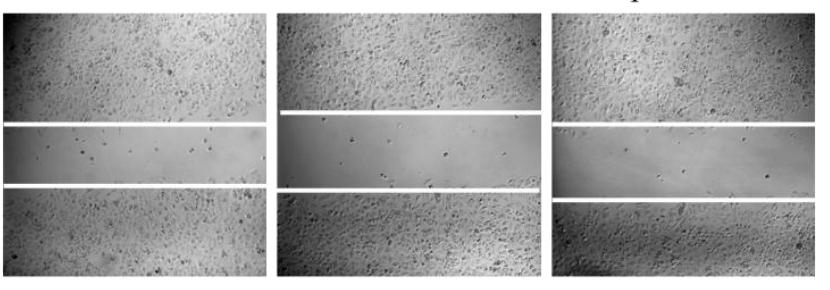

\section{$\mathrm{E}$}

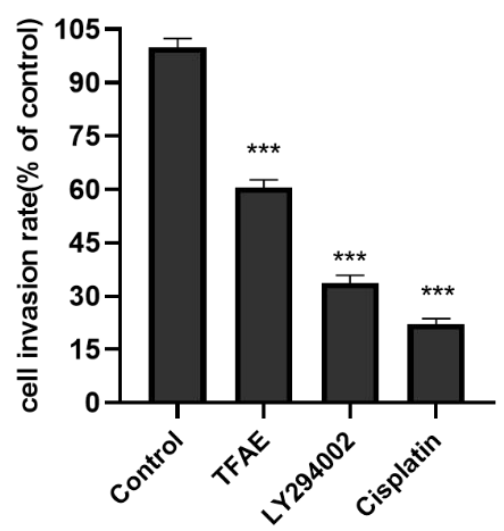

$\mathrm{F}$

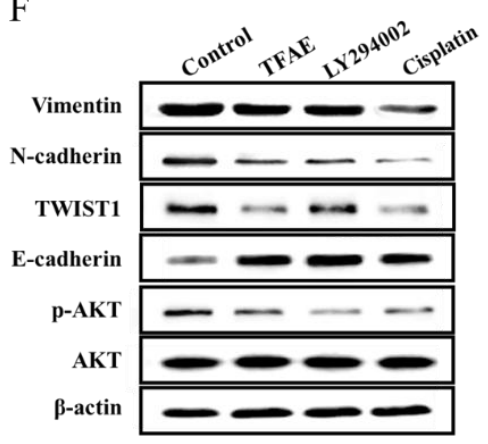

FIGURE 2 | TFAE inhibits PI3K/Akt pathway in EMT process of A549 cells. (A) Intersection protein targets of TFAE and lung cancer. 65 TFAE targets and 22474 lung cancer targets share 62 proteins. (B) Protein-Protein-Interaction (PPI) network of 62 proteins. AKT1, TP53, VEGFA, JUN show more connections with other proteins. (C) KEGG Pathway analysis of 62 proteins. $P<0.01$ was the cutoff for displaying the pathways. (D) The inhibition of PI3K/Akt-suppression and TFAE on wound healing. (E) The inhibition of PI3K/Akt-suppression and TFAE on A549 cell invasion. (F) The influence of PI3K/Aktsuppression and TFAE on the expression of EMT-related proteins. Statistical differences were determined by a two-sided Student's t-test. Compared with control, N.S. not significant, ${ }^{* \star *} P<0.001$; ${ }^{\star *} P<0.01 ;{ }^{*} P<0.05$. 


\subsection{TFAE inhibits TWIST1 in EMT process of A549 cells}

To identify the key target of TFAE, Cytoscape 3.6.1 was used to build "Ingredient-Target- Pathway" network. Among proteins involved in the network, TWIST1 attracted our attentions, which connected with both baicalein and wogonin (Figure 3A). To investigate whether TWIST1 contributes to the metastatic ability of A549 cells and whether TFAE affects the EMT process of A549 cells by inhibiting TWIST1, we applied small interfering RNA (siRNA) method to knock down the TWIST1 gene in A549 cell.

Compared with normal cells, TWIST1-silenced A549 cells showed significant lower mRNA level and protein expression (Figure 3B, C). Compared with the normal group after 24h, TFAE-treated/ TWIST1-silenced/TFAE\&siTWIST1-treated cells showed poor migration and invasion capabilities, and the simultaneous use of TFAE and siTWIST1 showed stronger inhibitory effect (Figure 3D, E). SiTWIST1-A549 cells showed increased expression of epithelial marker(E-cadherin) and decreased expression of mesenchymal markers (Vimentin and N-cadherin), as well as decreased level of EMTrelated transcription factor (TWIST1) and protein in PI3K/Akt pathway(p-AKT) (Figure 3F). TFAE showed similar effects to siTWIST1, and the synergistic application of TFAE and siTWIST1 showed more obvious effects (Figure 3F). Therefore, we speculate TFAE inhibits the EMT process of A549 cells by inhibiting TWIST1.
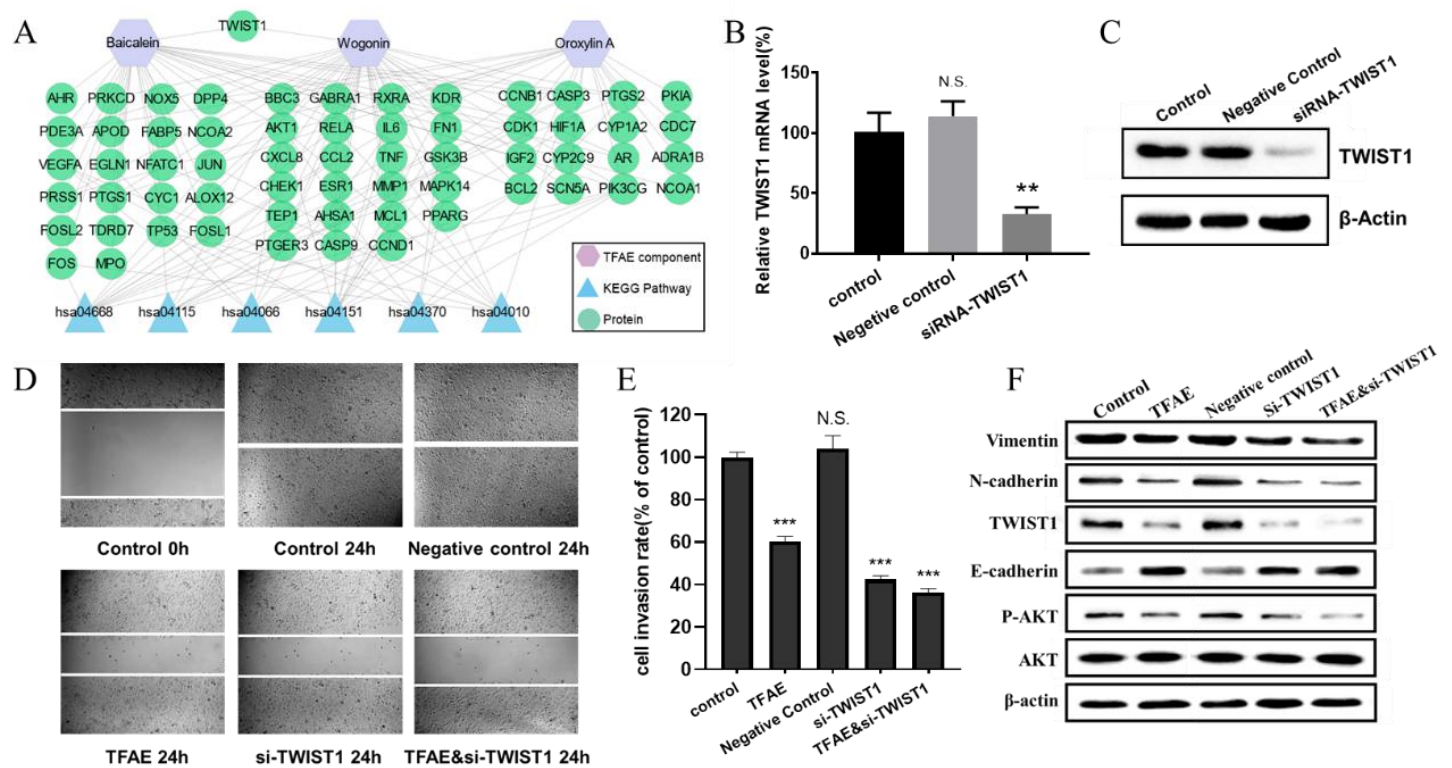

FIGURE 3 | TFAE inhibits TWIST1 in EMT process of A549 cells. (A)Relationships of 3 TFAE Components- 6 KEGG Pathways-62 Proteins. TWIST1 is a common protein target of baicalein and wogonin. (B)Relative TWIST1 mRNA level after siRNA interference. (C)Relative TWIST1 expression after siRNA interference. (D)The inhibition of siRNA-TWIST1 and TFAE on wound healing. (E)The inhibition of siRNA-TWIST1 and TFAE on A549 cell invasion rate. (F)The influence of siRNA-TWIST1 and TFAE on the expression of EMT-related proteins. Statistical differences were determined by a twosided Student's t-test; Compared with control, N.S., not significant, ${ }^{* * *} P<0.001 ;{ }^{* *} P<0.01 ;{ }^{*} P<0.05$.

\subsection{The effect of TFAE on protein expression of A549 cells}


We found that TFAE inhibited PI3K/Akt pathway and TWIST as above, yet it is not clear what happened that led to the decline of EMT. To explain the further mechanism, we collected A549 cells treated with TFAE for 24 hours and performed stable-isotope dimethyl labeling proteomics experiment. The proteins of A549 cells treated with DMSO or TFAE were marked with "Light" or "Heavy", respectively, then mixed, desalted and analyzed by LC-MS/MS (Figure 4A). Proteins with Average Ratio Light/ Heavy $(\mathrm{L} / \mathrm{H})>1.5$ were filtered out, and 158 proteins down-regulated by TFAE appeared at least twice in repeated experiments (Figure 4B). KEGG pathway analysis was performed on the 158 proteins and TFAE down-regulated pathways with $\mathrm{P}<0.01$ were as follows: Carbon metabolism, Glycolysis/Gluconeogenesis, Biosynthesis of amino acid and Pentose phosphate pathway (Figure 4C). These four pathways contain 11, 9, 7, and 4 proteins respectively (Table 1). In short, TFAE interferes with the sugar metabolism of A549 cells.

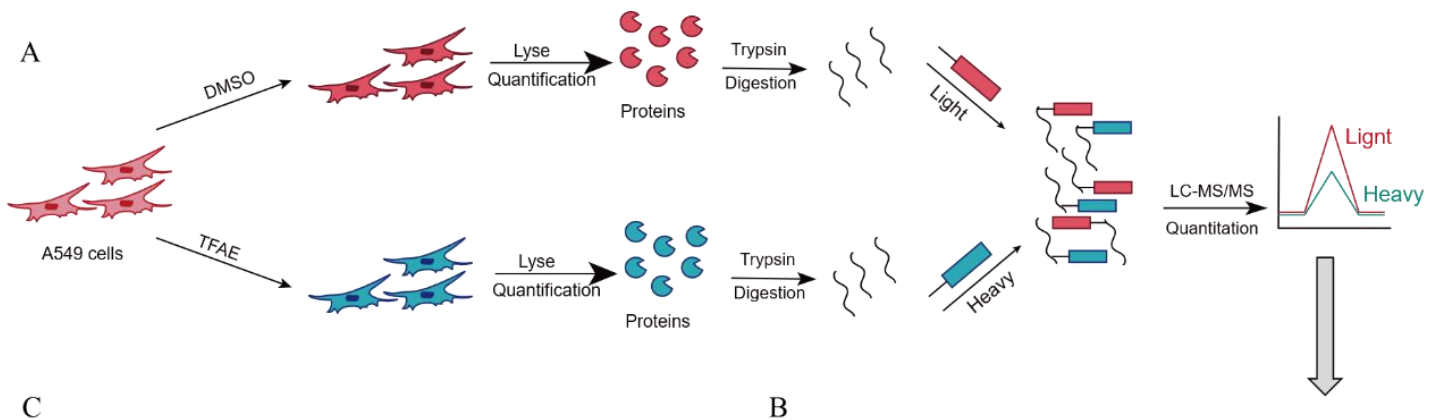

C TFAE down-regulated KEGG Pathway

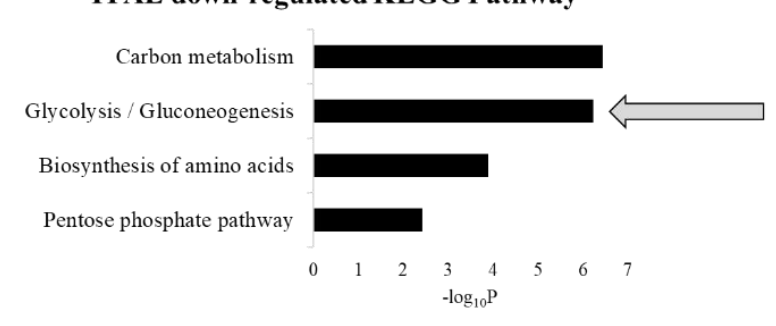

B
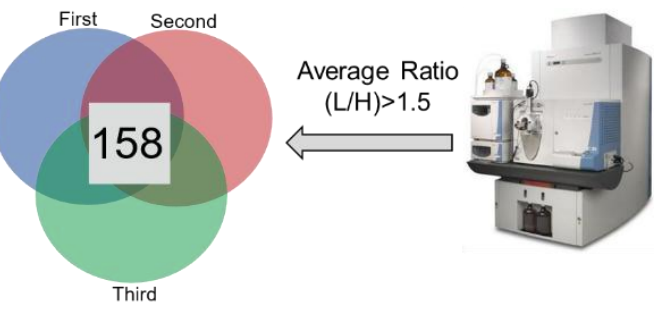

FIGURE 4 | TFAE interferes with the glucose metabolism of A549 cells. (A) Overall scheme of In-solution dimethyl labeling experiment, the control or TFAE group were labeled with "light" or "heavy" respectively. (B) Venn diagram showing the 158 proteins with an averaged ratio $>1.5$ appeared twice of triple experiment. (C) KEGG Pathway analysis of the 158 proteins. $P<0.01$ was the cutoff for displaying the pathways.

\section{Discussion}

The total flavonoids of Scutellaria baicalensis extract has an inhibitory effect on the EMT process of A549 cells, and may serve as an important breakthrough in the treatment of NSCLC(Yang et al., 2016). In this study, we configured TFAE according to the ratio of the main components and found that it may inhibit the EMT process of A549 cells by affecting PI3K/Akt pathway and TWIST1. In detail, firstly, we analyzed the possible mechanism of TFAE's effect on lung cancer and focused on PI3K/Akt pathway. The inhibition of LY294002 on PI3K/Akt pathway suppressed the migration and invasion of A549 cells, and caused high expression of epithelial-related protein and low expression of mesenchymal-related proteins. Secondly, network pharmacology analysis on the therapeutic effect of TFAE on lung cancer showed that TWIST1 was the common target of baicalein and wogonin. 
TWIST1 had been extensively studied in the EMT process, as shown below: the TWIST1-related axis may participate in the EMT process by activating the $\mathrm{Wnt} / \beta$-catenin signaling pathway, thereby accelerating the process of lung cancer(Pan et al., 2020). TWIST1 promotes EMT and metastasis through serine phosphorylation of p38, c-Jun N-terminal kinase (JNK) and Erk1/2(Jun et al., 2011). The flavonoids atalantraflavone targets to and reduces the stability of TWIST1, thereby inhibiting the process of NSCLC(Yuan et al., 2019). Therefore, TWIST1 is an important mediator in the process of cancer. TWIST1-targeted flavonoid provided a new strategy to inhibit EMT progress. In this study, "knockdown TWIST1" and "TFAE incubation" showed similar EMT-inhibition tendency, and the combined effect was more significant. In summary, we believe that TFAE inhibits PI3K/Akt pathway and TWIST1, thereby inhibiting the EMT process of A549 cells.

Stable-isotope dimethyl labeling proteomics results showed that TFAE down-regulated the carbon metabolism, glycolysis/gluconeogenesis, biosynthesis of amino acid, pentose phosphate pathway, etc. Tumor cells obtain energy mainly through the process of glycolysis, which promotes the EMT process of tumor cells(Liberti and Locasale, 2016); when nutrients were depleted, cancer cells tend to obtain metabolic materials through the pentose phosphate pathway(Ilias et al., 2020). Studies had shown that TWIST1 can promote glycolysis process(Li et al., 2015; Sara et al., 2020;Wang et al., 2020). In this study, ALDOA and GPI were enriched in both glycolysis and pentose phosphate pathways, while PKM and LDHA in glycolysis (Table 1). The relationships between the above genes and the EMT process had been reported: PKM expresses pyruvate kinase and catalyzes the transfer of phosphorylation groups from phosphoenolpyruvate to ADP to generate ATP and pyruvate(Dombrauckas et al., 2005). PKM is expressed in fetal tissues and cancers, and participates in the EMT process of human colon cancer cells (Wong et al., 2015). PKM interacts with TGF $\beta$ induced factor homeobox 2 (TGIF2) to inhibit the transcription of E-Cadherin(Hamabe et al., 2014). Glucose-6-phosphate isomerase edited by GPI catalyzes the conversion of glucose-6-phosphate to fructose-6-phosphate. GPI can also be produced by cancer cells to promote the EMT process(Y et al., 1998). GPI promotes the EMT process of breast cancer cells by inhibiting miR-200 and inducing ZEB1/2(Tatsuyoshi et al., 2009). Silencing GPI promotes the transition of human lung fibroblasts from the mesenchymal to the epithelial state(Tatsuyoshi et al., 2007).ALDOA expresses fructosebisphosphate aldolase A, which is involved in glycolysis and gluconeogenesis, and is also overexpressed in cancer(Yu-Chan et al., 2018). ALDOA is highly expressed in lung squamous cell carcinoma (LSCC) and depletion of ALDOA in lung squamous carcinoma cells reduces cell motility capabilities (Du et al., 2014). Overexpression of ALDOA in colon cancer cells leads to the EMT progress(Feng et al., 2018).In pancreatic cancer and bladder cancer cells, ALDOA-silencing increases E-Cadherin and decrease N-Cadherin expression(Ji et al., 2016; Jianwei et al., 2019). Lactate dehydrogenase-A (LDHA) enzyme converts pyruvate into lactic acid. LDHA promotes the EMT process, but the specific mechanism is not clear(Hou et al., 2019; Tirpe et al., 2019).

In summary, TFAE can inhibit the PI3K/Akt pathway with the core of TWIST1, thereby inhibiting the glycolytic pathway to suppress EMT in A549 cells (Figure 5). However, this study has limitations. Firstly, tumor cells are able to adapt to drug interventions, for example, cisplatin can be resistant in the treatment of NSCLC(Yuan et al., 2019). Therefore, whether TFAE has a long-lasting effect on NSCLC needs further study. Secondly, whether TFAE has a direct relationship with glycolysis or PI3K/AKT-related proteins needs further verification. Thirdly, all experiments were carried out in cells, next we will perform animal experiments. Moreover, we also explored the effects of the three main components of TFAE-baicalein, wogonin, and oroxylin-A on the EMT process, and found that baicalein had the most significant inhibitory effect on the EMT process of A549 cells, 
closing to the effect of TFAE. Next, we will explore the inhibitory mechanism of baicalein on NSCLC.

TABLE 1 | Pathways interfered by TFAE, and proteins enriched in each pathway

\begin{tabular}{ll}
\hline Pathways & Enriched Targets \\
\hline Carbon metabolism & GPI, PGAM1, PKM, HADHA, TALDO1, GAPDH, PGK1, MDH2, TPI1, \\
& ALDOA, G6PD \\
Glycolysis/Gluconeogenesis & GPI, PGAM1, PKM, LDHA, GAPDH, PGK1, TPI1, ALDH3A1, ALDOA \\
Biosynthesis of amino acid & PGAM1, PKM, TALDO1, GAPDH, PGK1, TPI1, ALDOA \\
Pentose phosphate pathway & GPI, TALDO1, ALDOA, G6PD \\
\hline
\end{tabular}

\section{Conflict of Interest}

The authors declare that the research was conducted in the absence of any commercial or financial relationships that could be construed as a potential conflict of interest.

\section{Author Contributions}

JD and HC designed all the experiments. HC, WZ and XX conducted most of the experiments. SS and CT designed siRNA primers. PD and FT participated in the analysis of network pharmacology. CJ, TF and SZ participated in the western blot experiment. JD performed LC-MS/MS analysis. All the authors participated in the manuscript writing.

\section{$8 \quad$ Funding}

This work is financially supported by the Science and Technology Project of Hebei Education Department (QN2018060) , National Key Research and Development Program of China (2018YFC1706300 and 2018YFC17063005), the Science and Technology Planning Project of Gansu Province (20JR10RA586), the Fundamental Research Funds for the Central Universities (lzujbky2019-92), the Open Project of State Key Laboratory of Natural Medicines (SKLNMKF202107), the Project for Longyuan Youth Innovation and Entrepreneurship Talent.

\section{Acknowledgments}

We thank North China University of Science and Technology and Lanzhou University for experimental platform. We thank the BioRxiv platform for allowing the article to be published in preprinted version.

\section{Reference styles}


(2020). 2020 World Health Statistics [Online]. International Agency for Research on Cancer. Available: https://www.who.int/data/collections [Accessed].

Ding, X., Li, F., and Zhang, L. (2019). Knockdown of Delta-like 3 restricts lipopolysaccharide-induced inflammation, migration and invasion of A2058 melanoma cells via blocking Twist1-mediated epithelialmesenchymal transition. Life Sci. 226, 149-155.

Dombrauckas, J.D., Santarsiero, B.D., and Mesecar, A.D. (2005). Structural basis for tumor pyruvate kinase M2 allosteric regulation and catalysis. Acta Crystallogr., Sect. A: Found. Crystallogr. 61, 9417-9429.

Dorota, W., Andrzej, D., and Adam, M. (2015). Antiradical and antioxidant activity of flavones from Scutellariae baicalensis radix. Nat. Prod. Res. 29, 1567-1570.

Du, S., Guan, Z., Hao, L., Song, Y., Wang, L., Gong, L., Liu, L., Qi, X., Hou, Z., and Shao, S. (2014). FructoseBisphosphate Aldolase A Is a Potential Metastasis-Associated Marker of Lung Squamous Cell Carcinoma and Promotes Lung Cell Tumorigenesis and Migration. PLoS One 9, e85804.

Eales, K.L., Hollinshead, K.E.R., and Tennant, D.A. (2016). Hypoxia and metabolic adaptation of cancer cells. Oncogenesis 5, e190.

Feng, Y., Yixing, C., Lu, X., Jiabian, L., and Shuyu, Y. (2018). Aldolase A overexpression is associated with poor prognosis and promotes tumor progression by the epithelial-mesenchymal transition in colon cancer. Biochem. Biophys. Res. Commun. 497, 639-645.

Gatenby, R.A., and Gillies, R.J. (2004). Why do cancers have high aerobic glycolysis? Nat. Rev. Cancer 4, 891-899.

Gongda, X., F, R.D., Qiang, L., Debby, H., Stephan, D., Daniel, H., Curzio, R., and A, H.B. (2012). Akt/PKBmediated phosphorylation of Twist1 promotes tumor metastasis via mediating cross-talk between PI3K/Akt and TGF- $\beta$ signaling axes. Cancer Discovery 2, 248-259.

Hamabe, A., Konno, M., Tanuma, N., Shima, H., Tsunekuni, K., Kawamoto, K., Nishida, N., Koseki, J., Mimori, K., Gotoh, N., Yamamoto, H., Doki, Y., Mori, M., and Ishii, H. (2014). Role of pyruvate kinase M2 in transcriptional regulation leading to epithelial-mesenchymal transition. Proc. Natl. Acad. Sci. 111, 15526-15531.

Heiden, M.G.V., Cantley, L.C., and Thompson, C.B. (2009). Understanding the Warburg Effect: The Metabolic Requirements of Cell Proliferation. Science 324, 1029-1033.

Hong, R., Peizhun, D., Zongyu, G., Yiting, J., Di, D., Xiuping, L., and Qiang, Z. (2016). TWIST1 and BMI1 in Cancer Metastasis and Chemoresistance. J. Cancer 7, 1074-1080.

Hou, X.-M., Yuan, S.-Q., Zhao, D., Liu, X.-J., and Wu, X.-A. (2019). LDH-A promotes malignant behavior via activation of epithelial-to-mesenchymal transition in lung adenocarcinoma. Biosci. Rep. 39, BSR20181476.

Ilias, G.-S., V, C.D., Venetsana, K., and Apostolos, Z. (2020). EMT Factors and Metabolic Pathways in Cancer. Front Oncol 10.

Jente, V.S., David, B., Peter, T.D., and Hans, V.D. (2018). Epithelial-mesenchymal-transition-inducing transcription factors: new targets for tackling chemoresistance in cancer? Oncogene 37, 6195-6211.

Ji, S., Zhang, B., Liu, J., Qin, Y., Liang, C., Shi, S., Jin, K., Liang, D., Xu, W., Xu, H., Wang, W., Wu, C., Liu, L., Liu, C., Xu, J., Ni, Q., and Yu, X. (2016). ALDOA functions as an oncogene in the highly metastatic pancreatic cancer. Cancer Lett. 374, 127-135.

Jianwei, L., Fang, W., Hongzhi, G., Sihuai, H., Fangzhen, C., and Jianguo, S. (2019). ALDOLASE A regulates invasion of bladder cancer cells via E-cadherin-EGFR signaling. J. Cell. Biochem., 13694-13705.

Jun, H., Jian, Z., Junjiang, F., Tao, H., Jun, Q., Li, W., Lan, L., and Jianming, X. (2011). Phosphorylation of serine 68 of Twist 1 by MAPKs stabilizes Twist1 protein and promotes breast cancer cell invasiveness. Cancer Res. 71, 3980-3990.

Keith, R.L. (2017). Lung carcinoma.

L, S.G. (2010). HIF-1: upstream and downstream of cancer metabolism. Curr. Opin. Genet. Dev. 20, 51-56.

Li, Y., Yixuan, H., Jie, Y., Shifu, T., Hailong, Z., Qing, Z., Yan-E, D., Mingli, Z., Siyang, W., Liyun, X., Xi, T., Xiaojiang, C., and Manran, L. (2015). Twist promotes reprogramming of glucose metabolism in breast cancer cells through PI3K/AKT and p53 signaling pathways. Oncotarget 6, 25755-25769.

Liberti, M.V., and Locasale, J.W. (2016). Correction to: 'The Warburg Effect: How Does it Benefit Cancer Cells?'. Trends Biochem. Sci. 41, 211-218.

Lopez-Lazaro, M. (2008). The Warburg Effect: Why and How Do Cancer Cells Activate Glycolysis in the Presence of Oxygen? Anti-Cancer Agents Med. Chem. 8, 305-312.

Mph, R.L.S., Mph, K.D.M., Dvm, A.J., and Phd (2017). Cancer Statistics, 2017. Ca-Cancer J. Clin. 67, 7-30.

Muz, B., Puente, P.D.L., Azab, F., and Azab, A.K. (2015). The role of hypoxia in cancer progression, angiogenesis, metastasis, and resistance to therapy. Hypoxia 2015, 83-92. 
Otsuki, Y., Saya, H., and Arima, Y. (2018). Prospects for new lung cancer treatments that target EMT signaling. Dev. Dyn. 247, 462-472.

P, C., P, J., K, R., S, J.J., H, P.H., and B, F.-R. (2000). Plasma concentrations of VCAM-1 and ICAM-1 are elevated in patients with Type 1 diabetes mellitus with microalbuminuria and overt nephropathy. Diabetic Med. 17, 644-649.

Pan, J., Fang, S., Tian, H., Zhou, C., Zhao, X., Tian, H., He, J., Shen, W., Meng, X., Jin, X., and Gong, Z. (2020). IncRNA JPX/miR-33a-5p/Twist1 axis regulates tumorigenesis and metastasis of lung cancer by activating Wnt/ß-catenin signaling. Mol. Cancer 19.

Qing-Qing, Z., Chenhui, M., Qian, W., Yong, S., and Tangfeng, L. (2016). The role of TWIST1 in epithelialmesenchymal transition and cancers. Tumor Biol. 37, 185-197.

Sara, L., Eliot, F., Hikaru, S., Janine, H., Sharmistha, L., Alexandre, H., Gangadhar, T., Erica, L., Rajan, D., Ignacio, R., Noritoshi, K., Changjiun, W., L, B.R., Nagireddy, P., Michael, Z., M, Z.E., S, L.V., and Raghu, K. (2020). Endothelial-to-mesenchymal transition compromises vascular integrity to induce Myc-mediated metabolic reprogramming in kidney fibrosis. Sci. Signaling 13, eaaz2597.

T, S.A., and Benjamin, S. (2011). Targeting anaplastic lymphoma kinase in lung cancer. Clin. Cancer Res. 17, 2081-2086.

Tatsuyoshi, F., Huankai, H., Takashi, Y., Victor, H., and Avraham, R. (2007). Down-regulation of phosphoglucose isomerase/autocrine motility factor results in mesenchymal-to-epithelial transition of human lung fibrosarcoma cells. Cancer Res. 67, 4236-4243.

Tatsuyoshi, F., Victor, H., and Avraham, R. (2009). Phosphoglucose isomerase/autocrine motility factor mediates epithelial and mesenchymal phenotype conversions in breast cancer. Cancer Res. 69, 53495356.

Tennant, D.A., Durán, R.V., and Gottlieb, E. (2010). Targeting metabolic transformation for cancer therapy. Nat. Rev. Cancer 10, 267-277.

Tieju, L., Xiulan, Z., Xu, Z., Yanjun, Z., Xueyi, D., Nan, Z., Shihan, L., and Baocun, S. (2020). The EMT transcription factor, Twist1, as a novel therapeutic target for pulmonary sarcomatoid carcinomas. Int. $J$. Mol. Sci. 56, 750-760.

Tirpe, A.A., Gulei, D., Ciortea, S.M., Crivii, C., and Berindan-Neagoe, I. (2019). Hypoxia: Overview on Hypoxia-Mediated Mechanisms with a Focus on the Role of HIF Genes. Int. J. Mol. Sci. 20, 6140.

Trott, O., and Olson, A.J. (2010). AutoDock Vina: Improving the speed and accuracy of docking with a new scoring function, efficient optimization, and multithreading. J. Comput. Chem. 31, 455-461.

Tulchinsky, E., Demidov, O., Kriajevska, M., Barlev, N.A., and Imyanitov, E. (2018). EMT: A mechanism for escape from EGFR-targeted therapy in lung cancer. BBA-Rev. Cancer 1871, 29-39.

Wang, X.-X., Yin, G.-Q., Zhang, Z.-H., Rong, Z.-H., Wang, Z.-Y., Du, D.-D., Wang, Y.-D., Gao, R.-X., and Xian, G.-Z. (2020). TWIST1 transcriptionally regulates glycolytic genes to promote the Warburg metabolism in pancreatic cancer. Exp. Cell Res. 386, 111713.

Wong, N., Ojo, D., Yan, J., and Tang, D. (2015). PKM2 contributes to cancer metabolism. Cancer Lett. 356, 184-191.

Y, N., S, P., A, H., H, W., and A, R. (1998). Expression and secretion of neuroleukin/phosphohexose isomerase/maturation factor as autocrine motility factor by tumor cells. Cancer Res. 58, 2667-2674.

Yang, W., Hui-Juan, C., Shu-Jun, S., Jian-Ye, D., Jun-Wei, F., Qian-Hua, L., Chao, Y., Wen-Wei, M., and Yong$\mathrm{Yu}, \mathrm{Z}$. (2016). Total flavonoid aglycones extract in Radix scutellariae inhibits lung carcinoma and lung metastasis by affecting cell cycle and DNA synthesis. J. Ethnopharmacol. 194, 269-279.

Yochum, Z.A., Cades, J., Wang, H., Chatterjee, S., Simons, B.W., Oâ Brien, J.P., Khetarpal, S.K., LemtiriChlieh, G., Myers, K.V., Huang, E.H.-B., Rudin, C.M., Tran, P.T., and Burns, T.F. (2019). Targeting the EMT transcription factor TWIST1 overcomes resistance to EGFR inhibitors in EGFR- mutant nonsmall-cell lung cancer. Oncogene 38, 656-670.

Yu-Chan, C., Yi-Chieh, Y., Chia-Ping, T., Chih-Jen, Y., and Michael, H. (2018). Roles of Aldolase Family Genes in Human Cancers and Diseases. Trends Endocrinol. Metab. 29, 549-559.

Yuan, T., Ling, F., Wang, Y., and Teng, Y. (2019). A natural product atalantraflavone inhibits non-small cell lung cancer progression via destabilizing Twist1. Fitoterapia 137, 104275.

\section{Data Availability Statement}

All datasets generated for this study are included in the manuscript and/or the supplementary files. 